\title{
Agradecimentos aos revisores 2020-2021
}

\section{Acknowledgement to Reviewers 2020-2021}

Os altos padrões científicos mantidos pela Revista Portuguesa de Investigação Comportamental e Social nos seus artigos deveram muito ao esforço dos revisores, que ofereceram livremente o seu tempo e conhecimento. Assim, os Editores da Revista Portuguesa de Investigação Comportamental e Social agradecem aos seguintes revisores que reviram artigos no período de novembro de 2020 a novembro de 2021.

The high scientific standards maintained by the Portuguese Journal of Behavioral and Social Research in its papers owe much to the effort of the reviewers, who gave their time and knowledge freely. Thus, the Editors of the Portuguese Journal of Behavioral and Social Research acknowledge with particular gratitude the following reviewers who have reviewed papers during the period November 2020-November 2021.

https://doi.org/10.31211/rpics.2021.7.2.241

\author{
Ana Cláudia Cardoso \\ Universidade Federal de Juiz de Fora; Instituto de Ciências \\ Humanas; Juiz de Fora, Brasil \\ André Freitas \\ Faculdade de Psicologia e de Ciências da Educação da \\ Universidade do Porto, Portugal

\section{Anne Daniele Nascimento} \\ Instituto Federal de Educação, Ciência e Tecnologia do Sudeste \\ de Minas Gerais, Campus Barbacena, Barbacena, Brasil

\section{Carla Susana Vicente} \\ Universidade de Évora, Portugal \\ Clara Cruz Santos \\ Faculdade de Psicologia e Ciência de Educação; \\ Universidade de Coimbra, Portugal
}

\section{Daniela Nogueira \\ Instituto Superior da Maia, Portugal}

Denise de Freitas

Universidade Federal de São Carlos, São Carlos, Brasil

\section{Diogo Carreiras}

Centro de Investigação em Neuropsicologia e Intervenção Cognitivo Comportamental, Universidade de Coimbra, Portugal

Fátima Regina Ney Matos (3X)

Instituto Superior Miguel Torga, Coimbra, Portugal

Filipe Rodrigues

Instituto Politécnico de Leiria, Portugal

\section{DI\&D | ISMT}

rpics@ismt.pt

https://rpics.ismt.pt
Gabinete editorial

Revista Portuguesa de Investigação

Instituto Superior Miguel Torga 


\section{Gabriela Jorge}

Centro de Estudos Sociais da Universidade de Coimbra; Instituto Superior Miguel Torga, Coimbra, Portugal

\section{Gonçalo Dias}

Universidade de Coimbra, Portugal

\section{Ilda Maria Massano-Cardoso}

Instituto Superior Miguel Torga; Faculdade de Medicina da Universidade de Coimbra, Portugal

Inês Amaral

Universidade de Coimbra, Portugal

Inês Pimentel

Instituto Superior Miguel Torga, Coimbra, Portugal

\section{Jairo Simião Dornelas}

Universidade Federal de Pernambuco, Brasil

Joana Maria Aleluia Gomes Sequeira

Instituto Superior Miguel Torga, Coimbra, Portugal

\section{José Carlos Carvalho}

Escola Superior de Enfermagem do Porto, Portugal

\section{José Carlos Rodrigues Gomes}

Escola Superior de Saúde do Instituto Politécnico de Leiria, Portugal

\section{José Mendes}

INTELECTO - Psicologia \& Investigação, Açores, Portugal

\section{Laura Susana Tavares Lemos}

Instituto Superior Miguel Torga, Coimbra, Portugal

\section{Luís Manuel Loureiro}

Escola Superior de Enfermagem de Coimbra, Portugal

\section{Luísa Pimentel}

Escola Superior de Educação e Ciências Sociais do Instituto Politécnico de Leiria, Portugal

\section{Madalena Alarcão}

Universidade de Coimbra, Portugal
Manuel José Lopes

Universidade de Évora, Portugal

\section{Margarida Tenente Santos Pocinho}

Escola Superior de Tecnologia da Saúde de Coimbra, Portugal

\section{Maria Helena Gomes dos Reis Amaro da Luz}

Universidade de Coimbra, Portugal

\section{Mariana Vaz Pires Marques}

Centro de Responsabilidade Integrado de Psiquiatria do Centro Hospitalar e Universitário de Coimbra, Portugal

\section{Marlene Rosa}

Escola Superior de Saúde, Instituto Politécnico de Leiria; Center for Innovative Care and Health Technology, Portugal

\section{Nathália Matoso de Vasconcelos}

Fundação Oswaldo Cruz, Brasil

\section{Paulo Queirós}

Escola Superior de Enfermagem de Coimbra, Portugal

\section{Rafael Cano Tenorio}

Universidad de Cádiz, Espanha

\section{Sara Guerra}

Universidade de Aveiro, Departamento de Educação e Psicologia, Portugal

\section{Sara Oliveira}

Centro de Investigação em Neuropsicologia e Intervenção Cognitivo-Comportamental; Universidade de Coimbra, Portugal

\section{Sónia Catarina Carvalho Simões (2X)}

Instituto Superior Miguel Torga, Coimbra, Portugal

\section{Sónia Guadalupe}

Instituto Superior Miguel Torga; Centro de Estudose Investigação em Saúde da Universidade de Coimbra, Portugal

\section{Sónia Ribeiro}

Universidade Lusófona de Humanidades e Tecnologias, Portugal

\section{Stela Lemos}

Universidade Federal de Minas Gerais, Brasil 